\section{The Detour Polynomials of Ladder Graphs}

Ali Aziz Ali

aliazizali1933@yahoo.com

Academic Professor

University of Mosul

\section{Received on: 01/08/2011}

Gashaw A. Muhammed-Saleh

gashaw.mohammed@su.edu.krd

College of Science

University of Salahaddin

\title{
ABSTRACT
}

Accepted on: 03/10/2011

The detour distance $D(u, v)$ between two distinct vertices $u$ and $v$ of a connected graph $G$ is the length of a longest $u-v$ path in $G$. The detour index $d d(G)$ of $G$ is defined by $\sum_{\{u, v\}} D(u, v)$, and the detour polynomial of $G$ is $D(G ; x)=\sum_{\{u, v\}} x^{D(u, v)}$. The detour indices and detour polynomials of some ladder graphs are obtained in this paper. Keywords: Detour distance, Detour index, Detour polynomials, Ladder graphs.

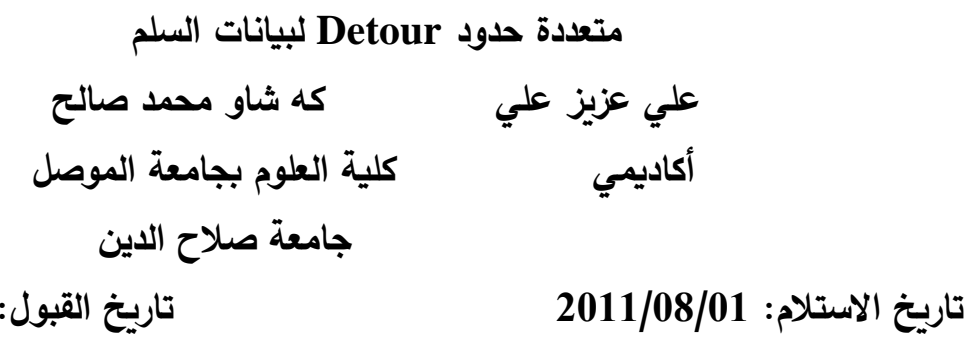

تاريخ القبول: 2011/10/03

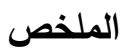

تعرف مسافة الالتقاف D(u,v) بين رأسين مختلفين u و v في بيان متصل G على أنها الطول لأطول

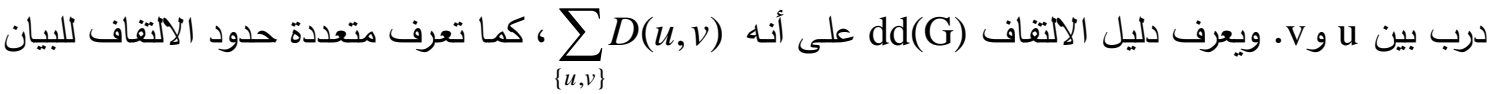
. $D(G ; x)=\sum_{\{u, v\}} x^{D(u, v)} \mathrm{G}$

تضمن هذا البحث إيجاد متعددة حدود الالتفاف ودليل الالتفاف لأنواع من البيانات المتصلة والتي هي بشكل

سُلم (Ladder).

الكلمات المفتاحية: مسافة Detour ، دليل Detour ، متعددة حدود Detour ، بيانات السلم

\section{Introduction}

For the definitions of graph concepts and notations see the books [1] and [7].

The detour distance $D(u, v)$ between two distinct vertices $u$ and $v$ in a connected graph $G$ is the maximum of the lengths of $u-v$ paths in $G$ (See [2, 3, 4, 5, 6 and 8]). A $u-v$ path of length $D(u, v)$ is called $\boldsymbol{u}-\boldsymbol{v}$ detour. As with standard distance, the detour distance $D$ is a metric on the vertex set $V(G)$ of any connected graph $G$. That is

(1) $D(u, v) \geq 0$ for all vertices $u, v \in V(G)$,

(2) $D(u, v)=0$ if and only if $u=v$,

(3) $D(u, v)=D(v, u)$ for all vertices $u$ and $v$ of $G$, and

(4) $D(u, v)+D(v, w) \geq D(u, w)$ for all vertices $u, v$ and $w$ of $G$.

It is clear that $D(u, v)=1$ if and only if $u v$ is a bridge of $G$, and $D(u, v)=p(G)-1$ if and only if $G$ contains a hamiltonian $u-v$ path. Moreover, 
$D(u, v)=d(u, v)$ for every two vertices $u$ and $v$ of $G$ if and only if $G$ is a tree. For other properties of the detour distance see [2 and 5].

The detour eccentricity $e_{D}(v)$ of a vertex $v$ in a connected graph $G$ is $e_{D}(v)=\max \{D(u, v): u \in V(G)\}$.

The detour radius $\operatorname{rad}_{D}(G)$ of a connected graph $G$ is defined as $\operatorname{rad}_{D}(G)=\min \left\{e_{D}(v): v \in V(G)\right\}$,

while the detour diameter $\operatorname{diam}_{D}(G)$ of $G$ is

$\operatorname{diam}_{D}(G)=\max \left\{e_{D}(v): v \in V(G)\right\}$.

In any connected graph $G$, the detour radius and detour diameter are related by the following inequalities[1]:

$\operatorname{rad}_{D}(G) \leq \operatorname{diam}_{D}(G) \leq 2 \operatorname{rad}_{D}(G)$.

The detour index $d d(G)$ of a connected graph $G$ is the Wiener index with respect to detour distance, that is

$d d(G)=\sum_{u, v} D(u, v)$

where the summation is taken over all unordered pairs of vertices $u$ and $v$ of $G$.

The detour distance of a vertex $v$, denoted by $d_{D}(v)$, is defined by

$\mathrm{d}_{\mathrm{D}}(\mathrm{v})=\sum_{\mathrm{u} \in \mathrm{V}(\mathrm{G})} \mathrm{D}(\mathrm{u}, \mathrm{v})$.

It is clear that

$d d(G)=\frac{1}{2} \sum_{v \in V(G)} d_{D}(v)$.

This index has recently received some attention in the chemical literature [9], because $d d(G)$ certainly carries some interesting structural information for cyclic compounds.

We introduce distance polynomial based on detour distance of a connected graph $G$ defined by

$D(G ; x)=\sum_{\{u, v\}} x^{D(u, v)}$,

where the summation is taken over all unordered pairs $u, v$ of distinct vertices of $G$. Such polynomial of $G$ will be called the detour polynomial (or detour distance polynomial) of $G$. It is clear that

$d d(G)=\left.\frac{d}{d x} D(G ; x)\right|_{x=1}$.

Moreover, one easily notice that

$D(G ; x)=\sum_{k=1}^{\delta_{D}} C_{D}(G, k) x^{k}$

where $\delta_{D}=\operatorname{diam}_{D}(G)$, and $C_{D}(G, k)$ is the number of unordered pairs of distinct vertices $u, v$ such that $D(u, v)=k$. The detour polynomial of a vertex $v$ of $G$ is defined as

$D(v, G ; x)=\sum_{\substack{u \in V(G) \\ u \neq v}} x^{D(v, u)}$.

It is clear that 


$$
D(G ; x)=\frac{1}{2} \sum_{v \in V(G)} D(v, G ; x),
$$

and

$$
D(v, G ; x)=\sum_{k \geq 1}^{e_{D}(v)} C_{D}(v, G ; k) x^{k},
$$

in which $C_{D}(v, G ; k)$ is the number of vertices $u(\neq v)$ such that $D(u, v)=k$ in $G$.

In this paper, we find detour polynomials and detour indices for a special class of graphs called ladders, namely $P_{n} \times K_{2}$ and Möbius ladder.

\section{The Detour Polynomial of a Ladder $L_{n}$ :}

A ladder $L_{n}$ is the graph $P_{n} \times K_{2}$, where $P_{n}$ is the $n$-path, $n \geq 3$. It is clear that $p\left(L_{n}\right)=2 n, q\left(L_{n}\right)=3 n-2$ and $\operatorname{diam}\left(L_{n}\right)=n$. Since $L_{n}$ is a hamiltonian graph, then $\operatorname{diam}_{D}\left(L_{n}\right)=2 n-1$.

The graph $L_{n}$ is shown in Fig. 2.1 with the vertices labeled $u_{1}, v_{1}, u_{2}, v_{2}, \ldots$, $u_{n}, v_{n}$.

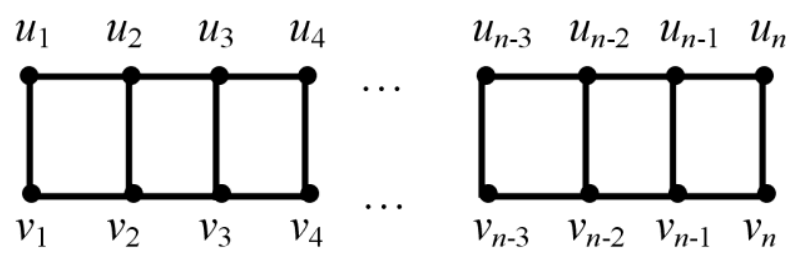

Fig. 2.1 The ladder $L_{n}, n \geq 4$

The following theorem determines the detour polynomial for $L_{n}, n \geq 4$.

\section{Theorem 2.1:}

For $n \geq 4$, we have

$$
\begin{aligned}
D\left(L_{n} ; x\right)=\left(n^{2}-n+2\right) x^{2 n-1}+\left(n^{2}-3 n+4\right) x^{2 n-2}+ & 2\left(x^{2}+x+1\right) \sum_{i=2}^{\left\lfloor\frac{n}{2}\right\rfloor} x^{2 n-2 i} \\
& + \begin{cases}0, & \text { for even } n, \\
2 x^{n+1}+x^{n}, & \text { for odd } n .\end{cases}
\end{aligned}
$$

\section{Proof: (I)}

First assume $n$ is even. From Fig.2.1, we find

$$
D\left(u_{1}, u_{j}\right)= \begin{cases}2 n-1, & \text { for even } j \geq 2, \\ 2 n-2, & \text { for odd } j \geq 3 .\end{cases}
$$

Also

$$
D\left(u_{1}, v_{j}\right)=\left\{\begin{array}{l}
2 n-1, \text { for odd } j \geq 1, \\
2 n-2, \text { for even } j \geq 2 .
\end{array}\right.
$$

Therefore, by the symmetry of $L_{n}$, we obtain

$$
D\left(w, L_{n} ; x\right)=n x^{2 n-1}+(n-1) x^{2 n-2}, w \in\left\{u_{1}, v_{1}, u_{n}, v_{n}\right\} \text {. }
$$

Now, for $i=2,3, \ldots, \frac{n}{2} ; i \neq j$ and $j \in\{1,2, \ldots, n\}$, we have 
$D\left(u_{i}, u_{j}\right)= \begin{cases}2 n-1, & \text { if }|j-i| \text { is odd } \\ 2 n-2, & \text { if }|j-i| \text { is even }\end{cases}$

Also, for $i=2,3, \ldots, \frac{n}{2}$ and $j \in\{1,2, \ldots, n\}-\{i, i-1, i+1\}$ we have

$D\left(u_{i}, v_{j}\right)= \begin{cases}2 n-1, & \text { if }|j-i| \text { is even, } \\ 2 n-2, & \text { if }|j-i| \text { is odd. }\end{cases}$

Finally, for $i=2,3, \ldots, \frac{n}{2}$ and $j=i+1$ or $i$ or $i-1$, we have

$D\left(u_{i}, v_{j}\right)=2 n- \begin{cases}2 i, & \text { if } j=i+1 \\ 2 i-1, & \text { if } j=i \\ 2 i-2, & \text { if } j=i-1\end{cases}$

Therefore, for $i=2,3, \ldots, \frac{n}{2}$, we have

$D\left(u_{i}, L_{n} ; x\right)=(n-1) x^{2 n-1}+(n-3) x^{2 n-2}+x^{2 n-2 i}+x^{2 n-2 i+1}+x^{2 n-2 i+2}$.

It is clear from the Fig. 2.1, that (2.3) holds for $v_{i}, u_{n+1-i}$ and $v_{n+1-i}$, where $i=2,3, \ldots, \frac{n}{2}$. Thus, for even $n \geq 4$, we have from (2.2) and (2.3)

$D\left(L_{n} ; x\right)=\frac{1}{2} \sum_{w \in V\left(L_{n}\right)} D\left(w, L_{n} ; x\right)$

$=\frac{1}{2}\left\{4\left(n x^{2 n-1}+(n-1) x^{2 n-2}\right)+4 \sum_{i=2}^{\frac{n}{2}}\left[(n-1) x^{2 n-1}+(n-3) x^{2 n-2}+x^{2 n-2 i}+x^{2 n-2 i+1}+x^{2 n-2 i+2}\right]\right\}$

$=\left(n^{2}-n+2\right) x^{2 n-1}+\left(n^{2}-3 n+4\right) x^{2 n-2}+2\left(x^{2}+x+1\right) \sum_{i=2}^{\frac{n}{2}} x^{2 n-2 i}$, for even $n$.

(II) If $n$ is odd, then using the steps used in proving even case, we get (2.2), and (2.3) for $i=2$ to $i=\frac{n-1}{2}$. Then, we add $2 D\left(u_{\frac{n+1}{2}}, L_{n} ; x\right)$ inside the brackets \{\} , where $D\left(u_{\frac{n+1}{2}}, L_{n} ; x\right)=(n-1) x^{2 n-1}+(n-3) x^{2 n-2}+2 x^{n+1}+x^{n}$.

This completes the proof of the theorem.

For $L_{2}$ and $L_{3}$, we obtain by direct calculation:

$$
\begin{aligned}
& D\left(L_{2} ; x\right)=4 x^{3}+2 x^{2}, \\
& D\left(L_{3} ; x\right)=8 x^{5}+6 x^{4}+x^{3} .
\end{aligned}
$$

\section{Corollary 2.2:}

For $n \geq 2$, we have

$d d\left(L_{n}\right)= \begin{cases}4 n^{3}-\frac{13}{2} n^{2}+7 n-4, & \text { for even } n, \\ 4 n^{3}-\frac{13}{2} n^{2}+7 n-\frac{7}{2}, & \text { for odd } n\end{cases}$

\section{Proof:}


Taking the derivative of $D\left(L_{n} ; x\right)$ with respect to $x$ at $x=1$, we get

$$
\begin{aligned}
d d\left(L_{n}\right)=\left(n^{2}-n+2\right)(2 n-1)+ & \left(n^{2}-3 n+4\right)(2 n-2)+6 \sum_{i=2}^{\left\lfloor\frac{n}{2}\right\rfloor}(1) \\
& +6 \sum_{i=2}^{\left\lfloor\frac{n}{2}\right\rfloor}(2 n-2 i)+ \begin{cases}0, & \text { for even } n, \\
3 n+2, & \text { for odd } n .\end{cases} \\
= & \left.4 n^{3}-11 n^{2}+19 n-10+6(2 n+1)\left(\frac{n}{2}\right\rfloor-1\right)-12 \sum_{i=2}^{2} i+ \begin{cases}0, & \text { for even } n, \\
3 n+2, & \text { for odd } n .\end{cases} \\
= & 4 n^{3}-11 n^{2}+19 n-10+\left\{\begin{array}{l}
3(2 n+1)(n-2)-6\left(\frac{n}{2}+2\right)\left(\frac{n}{2}-1\right), \text { for even } n, \\
3(2 n+1)(n-3)-\frac{3}{2}\left(n^{2}-9\right)+3 n+2, \text { for odd } n .
\end{array}\right.
\end{aligned}
$$

Simplifying the expression we get (2.5).

\section{Remark:}

We notice that the polynomial $D\left(L_{n} ; x\right)$ is of degree $2 n-1$, and has $n$ zeros, with nonzero coefficients $a_{i}$ of the terms $a_{i} x^{i}, i=2 n-1, \ldots, n$.

\section{The Detour Polynomial of a Möbius Ladder:}

A Möbius ladder, denoted $M L_{n}$ is a ladder $L_{n}$ with the two edges $u_{1} v_{n}$ and $v_{1} u_{n}$ as shown in Fig.3.1. It is clear that $p\left(M L_{n}\right)=2 n, q\left(M L_{n}\right)=3 n, \operatorname{diam}\left(M L_{n}\right)=\left\lceil\frac{n}{2}\right\rceil$ and $\operatorname{diam}_{D}\left(M L_{n}\right)=2 n-1$

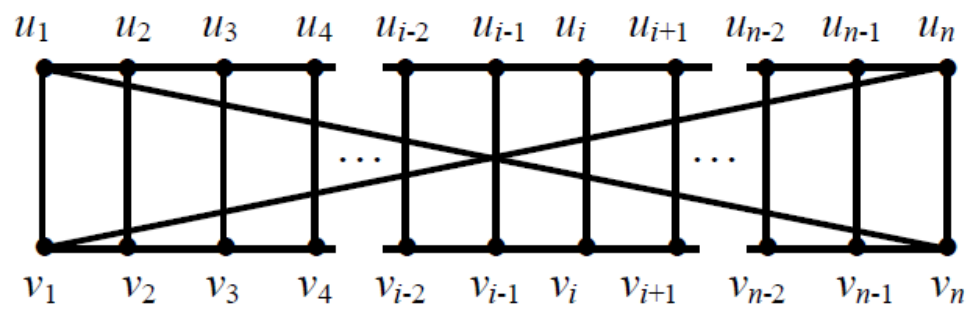

Fig.3.1 A Möbius ladder $M L_{n}, n \geq 5$

The graph $M L_{n}$ is a cubic hamiltonian graph and it can be redrawn as shown in Fig.3.2 from which we see that all its vertices have the same detour polynomial. Thus $D\left(M L_{n} ; x\right)=n D\left(u_{1}, M L_{n}\right.$;

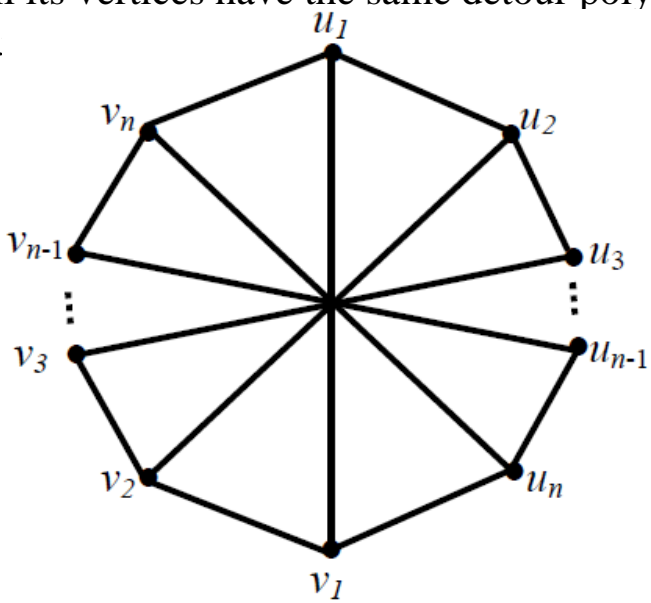

Fig.3.2 $M L_{n}, n \geq 5$ 
The detour polynomial of the Möbius ladder is obtained in the next theorem.

\section{Theorem 3.1:}

For $n \geq 2$,

$$
D\left(M L_{n} ; x\right)= \begin{cases}n(2 n-1) x^{2 n-1}, & \text { for even } n, \\ n^{2} x^{2 n-1}+n(n-1) x^{2 n-2}, & \text { for odd } n .\end{cases}
$$

\section{Proof:}

Assume that $n \geq 5$. We shall consider two cases for $n$.

(I) $n$ is even.

If $i$ is even, $i \geq 2$, then there is a hamiltonian $u_{1}-u_{i}$ path in $M L_{n}$, namely:

$u_{1}, v_{1}, v_{2}, u_{2}, u_{3}, v_{3}, \ldots, u_{i-1}, v_{i-1}, v_{i}, v_{i+1}, \ldots, v_{n}, u_{n}, u_{n-1}, \ldots, u_{i+1}, u_{i}$ (See Fig.3.1).

Thus

$D\left(u_{1}, u_{i}\right)=2 n-1, \quad i=2,4, \ldots, n$.

If $i$ is odd, $i \geq 3$, there is also a hamiltonian $u_{1}-u_{i}$ path in $M L_{n}$, namely:

$u_{1}, u_{2}, \ldots, u_{i-1}, v_{i-1}, v_{i-2}, \ldots, v_{2}, v_{1}, u_{n}, v_{n}, v_{n-1}, u_{n-1}, u_{n-2}, v_{n-2}, v_{n-3}, \ldots, v_{i}, u_{i}$.

Therefore

$D\left(u_{1}, u_{i}\right)=2 n-1$, for $i=3,5, \ldots, n-1$.

Now, we determine $D\left(u_{1}, v_{i}\right)$. If $i$ is even, $i \geq 2$, then there is a hamiltonian $u_{1}-v_{i}$ path, namely:

$u_{1}, u_{2}, \ldots, u_{i-1}, v_{i-1}, v_{i-2}, v_{i-3}, \ldots, v_{2}, v_{1}, u_{n}, v_{n}, v_{n-1}, u_{n-1}, u_{n-2}, v_{n-2}, v_{n-3}, \ldots, u_{i}, v_{i}$.

Thus

$D\left(u_{1}, v_{i}\right)=2 n-1$, for $i=2,4, \ldots, n$.

If $i$ is odd, $i \geq 1$, then there is a hamiltonian $u_{1}-v_{i}$ path, namely (this is for $i \geq 3$, for $i=1$, it is clear):

$u_{1}, v_{1}, v_{2}, u_{2}, u_{3}, \ldots, v_{i-1}, u_{i-1}, u_{i}, u_{i+1}, \ldots, u_{n}, v_{n}, v_{n-1}, v_{n-2}, \ldots, v_{i+1}, v_{i}$.

Thus

$D\left(u_{1}, v_{i}\right)=2 n-1$, for $i=1,3,5, \ldots, n-1$.

Hence, for every vertex $w\left(\neq u_{1}\right)$ of $M L_{n}$, we have $D\left(u_{1}, w\right)=2 n-1$.

Thus, from (3.1), we obtain (3.2) for even $n$.

(II) $n$ is odd.

Suppose that $i$ is even, then it is clear from Fig.3.1, that

$u_{1}, v_{1}, v_{2}, u_{2}, u_{3}, v_{3}, \ldots, u_{i-1}, v_{i-1}, v_{i}, v_{i+1}, \ldots, v_{n}, u_{n}, u_{n-1}, u_{n-2}, \ldots, u_{i}$,

is a hamiltonian $u_{1}-u_{i}$ path for even $i$. Thus

$D\left(u_{1}, u_{i}\right)=2 n-1$, for $i=2,4, \ldots, n-1$.

If $i$ is odd, then

$u_{1}, v_{1}, v_{2}, u_{2}, u_{3}, v_{3}, \ldots, u_{i-2}, v_{i-2}, v_{i-1}, v_{i}, \ldots, v_{n}, u_{n}, u_{n-1}, u_{n-2}, \ldots, u_{i}$ (which does not contain $u_{i-1}$ ) is a $u_{1}-u_{i}$ detour of length $2 n-2$, for odd $i$. Thus

$D\left(u_{1}, u_{i}\right)=2 n-2$, for $i=1,3,5, \ldots, n$.

To find $D\left(u_{1}, v_{i}\right)$, first assume $i$ is even, then

$u_{1}, v_{1}, v_{2}, u_{2}, u_{3}, v_{3}, \ldots, v_{i-2}, u_{i-2}, u_{i-1}, u_{i}, \ldots, u_{n}, v_{n}, v_{n-1}, v_{n-2}, \ldots, v_{i}$ (which does not contain $v_{i-1}$ ) is a $u_{1}-v_{i}$ detour of length $2 n-2$ for even $i$. Thus 
$D\left(u_{1}, v_{i}\right)=2 n-2$, for $i=2,4, \ldots, n-1$.

Now, let $i$ be odd, then there is a hamiltonian $u_{1}-v_{i}$ path

$u_{1}, v_{1}, v_{2}, u_{2}, u_{3}, v_{3}, \ldots, v_{i-1}, u_{i-1}, u_{i}, u_{i+1}, \ldots, u_{n}, v_{n}, v_{n-1}, v_{n-2}, \ldots, v_{i}$.

Thus

$D\left(u_{1}, v_{i}\right)=2 n-1$, for $i=1,3,5, \ldots, n$.

From (3.7) and (3.10) we get $n$ pairs of vertices $\left(u_{1}, u_{i}\right)$ and $\left(u_{1}, v_{i}\right)$ of detour distance $2 n-1$; and from (3.8) and (3.9), we get $(n-1)$ pairs of vertices $\left(u_{1}, u_{i}\right)$ and $\left(u_{1}, v_{i}\right)$ of detour distance $2 n-2$. Thus, from (3.1) we obtain (3.2) for odd $n$.

By direct calculation one may easily obtain:

$$
\begin{aligned}
& D\left(M L_{2} ; x\right)=6 x^{3}, \\
& D\left(M L_{3} ; x\right)=9 x^{5}+6 x^{4}, \\
& D\left(M L_{4} ; x\right)=28 x^{7}, \\
& D\left(M L_{5} ; x\right)=25 x^{9}+20 x^{8},
\end{aligned}
$$

which are the same results obtained from (3.2). Thus, the Theorem 3.1 holds for all values of $n \geq 2$.

From Theorem 3.1 and using (1.9) we get $d d\left(M L_{n}\right)$ as given in the next corollary:

\section{Corollary 3.2:}

For $n \geq 2$, the detour index of $M L_{n}$ is $d d\left(M L_{n}\right)= \begin{cases}n(2 n-1)^{2}, & \text { for even } n, \\ 4 n^{3}-5 n^{2}+2 n, & \text { for odd } n\end{cases}$

The following corollary is a useful graph theoretical result.

\section{Corollary 3.3:}

The Möbius ladder $M L_{n}$ is hamiltonian-connected if and only if $n$ is even.

A connected graph $G$ of order $p$ is called saturated (with respect to detour distance) [9] if $d d(G)=\frac{1}{2} p(p-1)^{2}$; that is if and only if $G$ is a hamiltonian-connected graph. Thus $M L_{n}$ is saturated if $n$ is even.

The density [9] of a $(p, q)$ connected graph $G$ is defined as $\operatorname{den}(G)=\frac{q}{p}$. One may show that the density of every saturated graph $G$ is not less that $\frac{3}{2}$. Thus, from corollary 3.3, $M L_{n}$ for even $n$, is saturated with minimum density $\frac{3}{2}$. 


\section{$\underline{\text { REFERENCES }}$}

[1] F. Buckley, and F. Harary (1990), Distance in Graphs, Addison-Wesley, Redwood City, California.

[2] G. Chartrand, H. Escuadro and P. Zhang (2005), Detour distance in graphs, J. Combin. Math. Combin. Comput., 53, 75-94.

[3] G. Chartrand, G. L. Johns and P. Zhang (2003), The detour number of a graph, Util. Math., 64, 97-113.

[4] G. Chartrand, G. L. Johns and P. Zhang, (2005) On the detour number and geodetic number of a graph, Ars. Combin.,34.

[5] G. Chartrand and P. Zhang (2004), Distance in graphs-taking the long view, J. Graphs. Combin., 1(1), 1-13

[6] G. Chartrand, G. L. Johns and S. Tian (1993), Detour distance in graphs, Annals of Discrete Maths., 55, 127-136.

[7] F. Harary (1969), Graph Theory, Addison-Wesley, Reading, Massachusetts.

[8] S. F. Kappor, H. V. Kronk and D. R. Link (1968), On detours in graphs, Canad. Math. Bull., 11, 195-201.

[9] M. Randic, L. M. DeAlba and F. E. Harris (1998), Graphs with the same detour matrix, Croatica Chemica Acta, CCACAA 71 (1), 53-68. 